\title{
Representation of the Basic Laws of Thermodynamics in Quantum Mechanics
}

\author{
Akira Suzuki ${ }^{1}$, Hisao Taira ${ }^{2}$ \\ ${ }^{1}$ Faculty of Science, Tokyo University of Science, Shinjuku-ku, Tokyo, Japan \\ ${ }^{2}$ Faculty of Education, Hokkaido University of Education, Kita-ku, Sapporo, Japan \\ Email: asuzuki@rs.kagu.tus.ac.jp, taira.hisao@s.hokkyodai.ac.jp
}

How to cite this paper: Suzuki, A. and Taira, H. (2018) Representation of the Basic Laws of Thermodynamics in Quantum Mechanics. Journal of Modern Physics, 9, 2420-2436.

https://doi.org/10.4236/jmp.2018.914155

Received: September 10, 2018

Accepted: December 11, 2018

Published: December 14, 2018

Copyright (C) 2018 by authors and Scientific Research Publishing Inc. This work is licensed under the Creative Commons Attribution International License (CC BY 4.0).

http://creativecommons.org/licenses/by/4.0/

(c) (i) Open Access

\begin{abstract}
We propose a representation of the basic laws, namely the zeroth, first, second and third law, in quantum thermodynamics. The zeroth law is represented by some parameters ( $\Theta$ 's) that specify respective quantum states. The parameters are the elements of thermodynamic state space $\mathcal{M}_{\Theta}$. The introduction of such parameters is based on a probabilistic nature of quantum theory. A quantum analog of the first law can be established by utilizing these parameters. The notion of heat in quantum systems is clarified from the probabilistic point of view in quantum theory. The representation of the second law can be naturally described in terms of these parameters introduced for the respective quantum systems. In obtaining the representation of quantum thermodynamics, consistency between quantum theory and classical thermodynamics should have been preserved throughout our formulation of quantum thermodynamics. After establishing the representation of the second law, the third law is discussed briefly. The relationship between thermodynamic temperatures and the parameters in $\mathcal{M}_{\Theta}$ is also discussed.
\end{abstract}

\section{Keywords}

Basic Laws of Thermodynamics, Thermodynamic State Space, Probabilistic Nature of Quantum Theory, Notion of Heat, Entropy Principle, Adiabatic Accessibility

\section{Introduction}

Thermodynamics is one of the theories which have high universality since thermodynamics as itself has been unchanged even if we now have a well-developed quantum theory. Classical thermodynamics has been well established by different approaches [1] [2] [3]. Lieb and Yngvason made the mathematical 
structure transparent by axiomatic approach [3]. Thermodynamics is a theory not only for classical but for quantum systems. Above all, the theoretical importance of thermodynamical consideration in quantum systems (quantum thermodynamics) is emphasized in text books [4] [5]. When we consider the thermodynamics for quantum systems, the important is the change in entropy since entropy is a constant of motion under the unitary transformation generated by a system Hamiltonian [6] [7]. A quantum heat engine has been investigated theoretically [8] [9]. Bender et al. studied a quantum Carnot cycle by considering a single quantum mechanical particle confined in a quantum well [9]. In their study, they found that the efficiency is equal to that of the Carnot cycle for classical case and proposed that the internal energy $U$ plays the same role as temperature. It should be however mentioned that in quantum system one cannot describe thermodynamic equibria in terms of a parameter like a temperature as in classical system [3].

This paper deals with the following questions that must be answered: Can thermodynamical laws refer to the variation of states of a system represented by the quantum states such as those states (eigenstates) of the Hamiltonian for a single quantum mechanical particle confined in a quantum well? If thermodynamical laws exist in quantum thermodynamic systems, how can they be expressed? To answer these questions, we need a representation which connects thermodynamic states and quantum states.

In classical thermodynamics, states are represented by points on a state space. A typical example of the state space is just a collection of $P$ (pressure) and $V$ (volume), i.e., a $P$ - $V$ plane. In quantum mechanics, what space should be used in order to describe thermodynamic states for quantum systems? In quantum mechanics, quantum (pure) states are expressed by the elements of a complex Hilbert space $\mathcal{H}$. However, the Hilbert space itself does not play the same role as the state space in classical thermodynamics since comparing one state vector with the others in $\mathcal{H}$ must be done by comparing the components of each vector. Thus we start with introducing a set $\mathcal{M}_{\Psi}$ of the state vectors in $\mathcal{H}$ in order to obtain a suitable set which plays the same role as the state space in classical thermodynamics. That is, we introduce a set $\mathcal{M}_{\Theta}$ which plays the same role as the state space and derive a correspondence between $\mathcal{M}_{\Psi}$ and $\mathcal{M}_{\Theta}$. After that, we will show that the first law of quantum thermodynamics can be described by the elements in $\mathcal{M}_{\Theta}$ and the internal energy of a quantum system can be described as a function on $\mathcal{M}_{\Theta}$. We will also discuss the relation between those parameters $\Theta$ 's in $\mathcal{M}_{\Theta}$ and thermodynamic temperatures.

The first law of thermodynamics is a law of conservation of energy and states the equivalence of heat and work. We will discuss the equivalence between work and heat in quantum thermodynamics. We assume that the energy of a system (i.e., the internal energy) is given by the expectation value of the Hamiltonian $\mathscr{H}: U=\langle\mathscr{C}\rangle=\sum_{i} p_{i} E_{i}$. In this expression, $E_{i}$ is the outcome of the expected energy state corresponding to a definite probability $p_{i}$ in a specified 
maximal test. Indeed, this probabilistic nature of quantum system plays a key role to establish a representation of the first law. Differentiating $U$ formally, we obtain the expression, $d U=\sum_{i}\left(E_{i} d p_{i}+p_{i} d E_{i}\right)$. The first term $\sum_{i} E_{i} d p_{i}$ implies there exists a non-mechanical source that induces a change in the internal energy of the system since a change in quantum states is in general determined by the unitary operator which does not change the definite probability (i.e., $\left.d p_{i}=0\right)$. The second term $\sum_{i} p_{i} d E_{i}$ implies a mechanical source that induces a change in the internal energy since we can trace the origin of $d E_{i}$ to an external parameter. As will be shown in Subsec. 3.2, the following identifications, $d^{\prime} Q=\sum_{i} E_{i} d p_{i}$ and $d^{\prime} W=\sum_{i} p_{i} d E_{i}$, are justified and are ensured by the existence of respective parameters $\Theta$ and $L$. We will show that the internal energy of quantum system is generally expressed in terms of parameters $\Theta$ and $L$, respectively, describing the equivalence relation among quantum states and external parameters. Therefore, the first law of quantum thermodynamics can be uniquely represented by these parameters. Once establishing the representation of the first law, it is worth to investigate the remaining thermodynamical laws (the second and third laws) for a quantum system described by quantum states.

In this paper, we propose a representation of the thermodynamical laws for quantum system in terms of the respective parameters and develop a theory of quantum thermodynamics based on the axiomatic theory of classical thermodynamics by Lieb and Yngvason [3]. In their formulation, the second law refers to the possible adiabatic transition of any two states in a state space.

This paper is organized as follows. In the next section, we present a brief review of classical thermodynamics. In Sec. 3, we state the basic notion of our formulation of quantum thermodynamics, and introduce a thermodynamic state space $\mathcal{M}_{\Theta}$ and a quantum state space $\mathcal{M}_{\Psi}$ and discuss the connection between them. In Subsec. 3.1 we show the existence of the zeroth law of quantum thermodynamics in the state space $\mathcal{M}_{\Theta}$. In Subsec. 3.2, the first law of thermodynamics and an adiabatic process are discussed. In Subsec. 3.3, we define entropy and give a representation of the second law, and discuss a relation among the adiabatic transitions, entropy and the term $d^{\prime} Q$. We refer to the third law in Subsec. 3.4. Finally, we give the results and discussion in Sec. 4.

\section{Classical Thermodynamics}

There are few approaches in thermodynamics [1] [2] [3]. Lieb and Yngvason's approach is helpful to understand the logical structure of thermodynamics. If thermodynamical laws exist in quantum systems as well as in classical systems, there must be the same logical structure in both systems. According to their formulation, a structure of adiabatic accessibility on a state space (thermodynamic state space) is characterized by an entropy inequality, i.e., the second law of thermodynamics. In this section we present a brief review of classical thermodynamics due to Lieb and Yngvason [3]. Thermodynamics is a theory which discusses a transition between equilibrium states. The second law 
refers to the feasible transitions in adiabatic process.

We start with introducing a formulation of the axiomatic thermodynamics proposed by Lieb and Yngvason [3]. In their formulation, the second law of thermodynamics is represented by the entropy principle.

Entropy principle: There is a real-valued function on all states of all systems (including compound systems), called entropy and denoted by $S$. Entropy has the following properties:

- Monotonicity: When $X$ and $Y$ are comparable states ${ }^{1}$, then

$$
X \prec Y \text { if and only if } S(X) \leq S(Y) \text {. }
$$

- Additivity: If $X$ and $Y$ are states of some (possibly different) systems and if $(X, Y)$ denotes the corresponding state in the composition of the two systems, then the entropy is additive for these states, i.e.,

$$
S(X, Y)=S(X)+S(Y) \text {. }
$$

- Extensivity: $S$ is extensive, i.e., for each $t>0$ and for each state $X$ and its scaled copy $t X$,

$$
S(t X)=t S(X) .
$$

It should be noted that entropy is determined by the physical (or thermodynamic) state of the system. In the entropy principle, $X$ and $Y$ (e.g., energy and volume) describe equilibrium states and are the elements of a state space (denoted by $\Gamma$ ). A system is then represented by the state space $\Gamma$ on which a relation " $\prec$ " of adiabatic accessibility is defined. The definition of adiabatic accessibility is as follows:

Adiabatic accessibility: A process whose only effect on the surroundings is exchange of energy with a mechanical source. This means that as a state arrives at new one, a state of surroundings is the same as before, in other words, the device returns to its initial state at the end of the process.

Lieb and Yngvason [3] proved that existence and uniqueness of entropy are equivalent to certain simple properties of a relation “ $\prec$ ” (A1 A6) and a comparison hypothesis $(\mathbf{C h})$ :

A1. Reflexivity: $X \stackrel{\mathrm{A}}{\sim} X$.

A2. Transitivity: $X \prec Y$ and $Y \prec Z$ implies $X \prec Z$.

A3. Consistency: $X \prec X^{\prime}$ and $Y \prec Y^{\prime}$ implies $(X, Y) \prec\left(X^{\prime}, Y^{\prime}\right)$.

A4. Scaling invariance: If $X \prec Y$, then $t X \prec t Y$ for $\forall t>0$.

A5. Splitting and Recombination: For $0<t<1, \quad X \stackrel{\mathrm{A}}{\sim}(t X,(1-t) X)$.

A6. Stability: If, for some pair of states, $X$ and $Y,\left(X, \epsilon Z_{0}\right) \prec\left(Y, \epsilon Z_{1}\right)$ holds for a sequence of $\epsilon$ 's tendency to zero and some states $Z_{0}$ and $Z_{1}$, then $X \prec Y$.

${ }^{1}$ The word "comparable" used in this paper means that any two states, $X$ and $Y$, in the same state space hold the relation, either $X \prec Y$ or $Y \prec X$, with respect to the relation “ $\prec$ ". In this context, $X$ and $Y$ are comparable and these states are called comparable states. 
Ch. Comparison hypothesis: The $\mathrm{Ch}$ holds for a state space $\Gamma$ if any two states $X$ and $Y$ on the space are comparable states, i.e., $X \prec Y$ or $Y \prec X$.

In the axiom $\mathbf{A 1}$, the symbol $\stackrel{\mathrm{A}}{\sim}$ denotes that two states $X$ and $Y$ are adiabatic equivalent; It describes a situation where both of the relations, $X \prec Y$ and $Y \prec X$, hold. It should be noted that the axioms, A3, A5 and A6, are defined on the product of state space $\Gamma \times \Gamma$, where $(X, Y) \in \Gamma \times \Gamma$. The Ch asserts that any two states on the same state space are comparable. Generally, the structure on the state space $\Gamma$ is determined by the axioms (A1 A6) and the comparison hypothesis $(\mathbf{C h})$ under the condition of adiabatic accessibility.

Let us consider a meaning of the entropy principle. Let $X, X^{\prime}, Y, Y^{\prime}, \cdots$ be the elements of the state space $\Gamma$. Imagine that we have a list of all possible pairs of states $X, Y$ such that $Y$ is adiabatic accessible from $X$. The foundation of thermodynamics and the essence of the second law are that this list, $X$ and $Y$, such as $X \prec Y$, can be simply encoded by the entropy function $S$ defined on a set of all states of systems (including compound systems). This means that $Y$ is adiabatic accessible from $X$, i.e., $X \prec Y$ if and only if $S(X) \leq S(Y)$ (entropy inequality). The entropy function should be kept consistency with the structure of the state space $\Gamma$ characterized by $\mathrm{A} 1 \sim \mathrm{A} 6$ and $\mathrm{Ch}$. Thus, we can characterize the structure based on the definition of adiabatic accessibility on the state space $\Gamma$ by using the entropy inequality. Combining the axioms (A1 A6) and the Equation (2), one can describe the entropy principle for systems including a compound system.

Let us consider a compound system in which $X, X^{\prime}$ and $Y, Y^{\prime}$ are the states of system $\mathrm{A}$ and system $\mathrm{B}$, respectively. In this case, the entropy principle is mathematically expressed as follows:

$$
(X, Y) \prec\left(X^{\prime}, Y^{\prime}\right) \text { if and only if } S(X)+S(Y) \leq S\left(X^{\prime}\right)+S\left(Y^{\prime}\right) \text {. }
$$

Note that all states $\left(X^{\prime}, Y^{\prime}\right)$ such that $X \prec X^{\prime}$ and $Y \prec Y^{\prime}$ are adiabatically accessible from $(X, Y)$. It is then important to notice that $\left(X^{\prime}, Y^{\prime}\right)$ can be adiabatically accessible from $(X, Y)$ even if $X^{\prime}$ is not adiabatically accessible from $X$. In such a case, entropy increase, $S\left(Y^{\prime}\right)-S(Y)$, in the process compensates for a loss, $S\left(X^{\prime}\right)-S(X)$, so as to satisfy the statement (4). Therefore, the inequality, $S(X)+S(Y) \leq S\left(X^{\prime}\right)+S\left(Y^{\prime}\right)$, characterizes the possible adiabatic transitions for the compound system even when $S(X) \geq S\left(X^{\prime}\right)$. It means that it is sufficient to know the entropy of each part of the compound system in order to decide which transition is feasible due to the interactions between the two subsystems.

For later use we write the entropy principle [the statement (4)] in terms of $U$ and $V$, where $U, V$ denote the internal energy and the volume of a system, respectively. Putting $X=\left(U_{\mathrm{A}}, V_{\mathrm{A}}\right)$ and $Y=\left(U_{\mathrm{B}}, V_{\mathrm{B}}\right)$, one obtains from the statement (4): 


$$
\begin{aligned}
& \left(\left(U_{\mathrm{A}}, V_{\mathrm{A}}\right),\left(U_{\mathrm{B}}, V_{\mathrm{B}}\right)\right) \prec\left(\left(U_{\mathrm{A}}^{\prime}, V_{\mathrm{A}}^{\prime}\right),\left(U_{\mathrm{B}}^{\prime}, V_{\mathrm{B}}^{\prime}\right)\right) \\
& \text { if and only if } S\left(U_{\mathrm{A}}, V_{\mathrm{A}}\right)+S\left(U_{\mathrm{B}}, V_{\mathrm{B}}\right) \leq S\left(U_{\mathrm{A}}^{\prime}, V_{\mathrm{A}}^{\prime}\right)+S\left(U_{\mathrm{B}}^{\prime}, V_{\mathrm{B}}^{\prime}\right) .
\end{aligned}
$$

It should be noted that the state of the compound system composed of system A and system $\mathrm{B}$ is described by $\left(U_{\mathrm{A}}, U_{\mathrm{B}}\right)$ only in the case where the volume is invariant during the process. The statement (5) makes sense in the case where $X$ is an extensive variable. However, there exists a particular case in which $X$ is an intensive variable.

One of the aims in this paper is to obtain a representation of entropy inequality for quantum system corresponding to the statement (4).

\section{Quantum Thermodynamics}

In order to obtain the representation of the zeroth, first, second, and third laws for quantum thermodynamics, we have to introduce a state space in order to describe thermodynamic states of quantum system. In the previous section, we have seen that the thermodynamic states of classical system denoted by capital Roman letters, $X, Y, Z$, etc. defined as the elements of state space $\Gamma$ satisfy certain simple properties of the relation " $\prec$ " $(\mathrm{A} 1 \sim \mathrm{A} 6)$ and the comparison hypothesis $(\mathrm{Ch})$. From the mathematical point of view, we expect that the thermodynamic structure of quantum thermodynamics should also have the same structure as that of classical thermodynamics.

In order to develop a representation of quantum thermodynamics, we must introduce a thermodynamic state space for quantum system since in quantum theory, quantum system is described by the complex Hilbert space $\mathcal{H}$ and the states of quantum system are in general described by the elements in $\mathcal{H}:\left|\Psi_{\alpha}\right\rangle$, $\left|\Psi_{\beta}\right\rangle$, etc. Here, Greek letter (subscript) denotes the label of respective states of quantum system. In the following, we use a symbol $\mathcal{M}_{\Psi}$ for quantum state space and a symbol $\mathcal{M}_{\Theta}$ (instead of $\Gamma$ in classical case) to represent thermodynamic state space for quantum system. Our aim in this section is to show the relation between $\mathcal{M}_{\psi}$ and $\mathcal{M}_{\Theta}$ in order to obtain the representation of quantum thermodynamics. To define the state space for quantum thermodynamics, namely thermodynamic state space in quantum systems, we have to establish the relation between $\mathcal{M}_{\Psi}$ and $\mathcal{M}_{\Theta}$. In this section we shall introduce a thermodynamic state space $\mathcal{M}_{\Theta}$ and obtain the representation of the zeroth law of quantum thermodynamics.

We first define a set $\mathcal{M}_{\Psi}$ as a quantum state space:

$$
\mathcal{M}_{\Psi}:=\left\{\left|\Psi_{\alpha}\right\rangle,\left|\Psi_{\beta}\right\rangle, \cdots\right\} .
$$

The set $\mathcal{M}_{\Psi}$ includes those elements which are linear combinations of the elements in $\mathcal{M}_{\Psi}$; e.g., $\left|\Psi_{\gamma}\right\rangle=\left|\Psi_{\epsilon}\right\rangle+c\left|\Psi_{\zeta}\right\rangle$, where $c$ is a complex number (a relative phase between $\left|\Psi_{\epsilon}\right\rangle$ and $\left|\Psi_{\zeta}\right\rangle$ ). The importance of this statement is well recognized in quantum theory [5]. All state vectors are thus found in the set $\mathcal{M}_{\psi}$ and they represent respective quantum states of the system. Now we would 
like to relate each element of thermodynamic states in $\mathcal{M}_{\Theta}$ to those state vectors in $\mathcal{M}_{\psi}$.

To find out the representation of the zeroth law associated with the property of $\mathrm{A} \mathbf{1}$ for quantum thermodynamics, we introduce a set $\mathcal{M}_{\Theta}$ as a thermodynamic state space. It should be noted that the elements in $\mathcal{M}_{\Theta}:=\left\{\Theta_{\alpha}, \Theta_{\beta}, \Theta_{\gamma}, \cdots\right\}$ have to be comparable each other in a context of the zeroth law of classical thermodynamics. As will be shown below, introducing these parameters $\Theta$ 's enables us to compare the quantum states in $\mathcal{M}_{\Psi}$ in thermodynamic sense.

We start with discussing the existence and uniqueness of $\Theta$ for quantum state $|\Psi\rangle$ representing a quantum system. Let us introduce the propositions of quantum theory, Q1 and Q2: [5].

Q1. A state is characterized by the probabilities of the various outcomes of every conceivable test.

Q2. If a quantum system is prepared in such a way that it certainly yields a predictable outcome in a specified maximal test ${ }^{2}$, the various outcomes of any other tests also have definite probabilities. In particular, these probabilities do not depend on the details of the procedure employed for preparing the quantum system. Therefore, the quantum system so prepared yields a specific outcome in the given maximal test. It should be noted that the quantum system prepared in such a way is said to be in a pure state.

Any complete orthogonal basis, $|i\rangle, i=1,2, \cdots, i, \cdots$, represents a realizable maximal test. Therefore one can obtain the definite probability $p_{i}$ for state $i$ from a probability amplitude: [10]

$$
a_{i}=\langle i \mid \Psi\rangle .
$$

It should be noted that the definite probability is given by $p_{i}=\left|a_{i}\right|^{2}$.

Now we introduce a lemma (L1) on the existence of parameter $\Theta_{j}$ $(\mathrm{j}=\alpha, \beta, \gamma, \cdots)$ :

L1. There exist parameters $\Theta_{\alpha}, \Theta_{\beta}, \cdots$ in $\mathcal{M}_{\Theta}$ for respective state vectors, $\left|\Psi_{\alpha}\right\rangle,\left|\Psi_{\beta}\right\rangle, \cdots$ in $\mathcal{M}_{\Psi}$.

Proof of L1. We treat a case for a label $\alpha$. Other cases, $\beta, \gamma, \cdots$, could be proved in the same way. From Equation (7), one can obtain a sequence $\left\{\left|a_{1}^{\alpha}\right|^{2},\left|a_{2}^{\alpha}\right|^{2}, \cdots,\left|a_{i}^{\alpha}\right|^{2}, \cdots\right\}$. By normalizing a state vector $\left|\Psi_{\alpha}\right\rangle$, the sequence satisfies the condition $\sum_{i=1}\left|a_{i}^{\alpha}\right|^{2}=1$ and it is clear that $0 \leq\left|a_{i}^{\alpha}\right|^{2} \leq 1$. Then each element of the sequence describes a definite probability for the respective state $i$. Hereafter, we shall omit superscript $\alpha$ for simplicity. Let us introduce a probability function $P(\Theta ; x)$ so as to satisfy $P(\Theta ; x=i)=\left|a_{i}\right|^{2}$ for any $i$. In

${ }^{2}$ Maximal test is defined as follows: Let $N$ (assumed to be finite for simplicity) be the maximum number of different outcomes obtainable in a test of a given quantum system. Then, any tests that have exactly $N$ different outcomes are called maximal (complete). Such tests are called maximal test. 
$P(\Theta ; x), \Theta$ is a parameter and $x$ is a random variable. Therefore, the parameter $\Theta_{\mathrm{j}}(\mathrm{j}=\alpha, \beta, \gamma, \cdots)$ exists for the representation of a state vector $\left|\Psi_{\mathrm{j}}\right\rangle$.

The L1 ensures the existence of parameters $\Theta$ 's which correspond to respective state vectors $\left|\Psi_{\mathrm{j}}\right\rangle$ 's in $\mathcal{M}_{\Psi}$ through probability functions $P\left(\Theta_{\mathrm{j}} ; x\right)$ 's. It is noted that a parameter is thereby found in each probability function for respective labels, $\mathrm{j}=\alpha, \beta, \gamma, \cdots$. Since Greek letters refer to the respective quantum states, $\left|\Psi_{\mathrm{j}}\right\rangle$ corresponds to $\Theta_{\mathrm{j}}(\mathrm{j}=\alpha, \beta, \gamma, \cdots)$. In $\mathrm{L} 1$, we saw that there exists unique parameters $\Theta$ 's assigned for every state vector in $\mathcal{M}_{\Psi}$. However, in order to represent the zeroth law in terms of $\Theta$ 's, the elements in $\mathcal{M}_{\Theta}$ must be mathematically comparable each other. Thus, it is required to utilize the same maximal test in order to obtain the equivalence relation for the element $\Theta_{j}$ from the corresponding equivalence relation for the state vector $\left|\Psi_{\mathrm{j}}\right\rangle$ for $\mathrm{j}=\alpha, \beta, \cdots$.

Adopting this requirement, it is ensured that those elements in $\mathcal{M}_{\Theta}$ are comparable each other and the $\mathrm{Ch}$ holds for $\Theta_{j}$ 's once entropy is defined as a function of $\Theta$. Therefore, the zeroth law of quantum thermodynamics can be uniquely established for quantum systems by making use of parameters $\Theta_{j}$ 's. In order to prove the existence of the zeroth law and to discuss the parameter $\Theta$, we have to introduce a proposition of quantum theory, Q3:

Q3. $|\Psi\rangle$ describes the same state as $c|\Psi\rangle$, where $c$ is a complex number.

Here, we discuss the parameter $\Theta$ associated with the proposition Q3. Let us introduce a map $f: \mathcal{M}_{\Psi} \mapsto \mathcal{M}_{\Psi}^{c}$, where $\mathcal{M}_{\Psi}^{c}$ is a set defined by $\mathcal{M}_{\Psi}^{c}:=\left\{c\left|\Psi_{\alpha}\right\rangle, c\left|\Psi_{\beta}\right\rangle, \cdots\right\}$. From the Q3, $\mathcal{M}_{\Psi} \equiv \mathcal{M}_{\Psi}^{c}$ since it is required that the set $\mathcal{M}_{\Psi}$ is invariant under the scalar transformation $f$. Therefore, the set $\mathcal{M}_{\Theta}$ is also invariant under the scalar transformation, i.e., $f: \mathcal{M}_{\Theta} \mapsto \mathcal{M}_{\Theta}^{c}$. This can be described in terms of their elements, i.e., $f: \Theta_{\mathrm{j}} \mapsto c \Theta_{\mathrm{j}} \quad(\mathrm{j}=\alpha, \beta, \gamma, \cdots)$. Thermodynamically, this means that the parameter $\Theta$ is an intensive variable. It should be noticed that the zeroth law is invariant under the scalar transformation of state vectors.

Let us derive the thermodynamic state space $\mathcal{M}_{\Theta}$ from $\mathcal{M}_{\Psi}$, where the elements in $\mathcal{M}_{\Theta}$ correspond to respective state vectors in $\mathcal{M}_{\Psi}$. It should be noted that in order to obtain the corresponding equivalence relation for these elements $\Theta_{\mathrm{j}}$ from the equivalence relation for $\left|\Psi_{\mathrm{j}}\right\rangle,(\mathrm{j}=\alpha, \beta, \gamma, \cdots)$, we have to use the same maximal test. In other words, when we compare the quantum states, those vectors in $\mathcal{M}_{\Psi}$ representing the states must be comparable and hence their components must be described by the same complete orthogonal basis. Adopting this requirement, it is ensured that the elements in $\mathcal{M}_{\Theta}$ are comparable each other and can specify the respective states of quantum system. Though $\mathcal{M}_{\Theta}$ should be written as $\mathcal{M}_{\Theta, L}$, where $L$ 
denotes a label of maximal test, $L$ will be omitted when we consider a fixed $L$ for the sake of simplicity.

Now one can compare two quantum states in thermodynamic sense since those parameters $\Theta$ 's can be used to describe two or more systems being equivalent. This leads to the zeroth law of quantum thermodynamics.

\subsection{The Zeroth Law of Quantum Thermodynamics}

Let us prepare three systems, $\mathcal{M}_{\Theta}^{\mathrm{A}}, \mathcal{M}_{\Theta}^{\mathrm{B}}$ and $\mathcal{M}_{\Theta}^{\mathrm{C}}$, where superscripts indicate the labels of respective systems. The equivalence relation among quantum states is described by

$$
\text { If } \Theta_{\alpha}^{\mathrm{A}}=\Theta_{\beta}^{\mathrm{B}} \text {, then }\left|\Psi_{\alpha}\right\rangle^{\mathrm{A}} \doteq\left|\Psi_{\beta}\right\rangle^{\mathrm{B}},
$$

where $\Theta_{\alpha}^{\mathrm{A}} \in \mathcal{M}_{\Theta}^{\mathrm{A}}$ and $\Theta_{\beta}^{\mathrm{B}} \in \mathcal{M}_{\Theta}^{\mathrm{B}}$, respectively. In this relation, a symbol $\dot{\doteq}$ means that the state in the left-hand side is equivalent to the state in the right-hand side. Later this relation (8) reaffirm the zeroth law of thermodynamics.

Proof of the zeroth law. Let $\Theta_{\alpha}^{\mathrm{A}}$ and $\Theta_{\beta}^{\mathrm{B}}$ be the elements of $\mathcal{M}_{\Theta}^{\mathrm{A}}$ and $\mathcal{M}_{\Theta}^{\mathrm{B}}$, respectively. By L1, it is clear that $\Theta_{\alpha}^{\mathrm{A}}=\Theta_{\beta}^{\mathrm{B}}$ implies $\left|\Psi_{\alpha}\right\rangle^{\mathrm{A}} \doteq\left|\Psi_{\beta}\right\rangle^{\mathrm{B}}$.

We are now in a position to discuss some consequences obtained by introducing the parameter $\Theta_{j}$ to specify the corresponding thermodynamic states of quantum systems. It is clear from Equation (8) that the transitiveness lawholds:

$$
\text { If } \Theta_{\alpha}^{\mathrm{A}}=\Theta_{\beta}^{\mathrm{B}} \text { and } \Theta_{\beta}^{\mathrm{B}}=\Theta_{\gamma}^{\mathrm{C}} \text {, then }\left|\Psi_{\alpha}\right\rangle^{\mathrm{A}} \dot{=}\left|\Psi_{\gamma}\right\rangle^{\mathrm{C}} \text {. }
$$

We have established a representation of the zeroth law of quantum thermodynamics (equivalence relation among quantum states). The zeroth law can be expressed in terms of parameters in $\mathcal{M}_{\Theta}$.

\subsection{The First Law of Quantum Thermodynamics}

The first law of thermodynamics is the law of conservation of energy and it assures equivalence between heat and work. It is written as

$$
d U=d^{\prime} Q+d^{\prime} W,
$$

where $d U$ is a small change in the internal energy of a system, $d^{\prime} Q$ is the heat transferred to the system and $d^{\prime} W$ is the work done on the system. Though Equation (10) is for classical system, we will show the same expression holds for quantum system.

We consider a quantum system described by Hamiltonian $\mathscr{K}$. Here we only assume that the internal energy of the system, $U$, is given by the pure state expectation value of Hamiltonian $\mathscr{H}::^{3}$

$$
U=\langle\mathscr{H}\rangle=\sum_{i} p_{i} E_{i},
$$

${ }^{3}$ The energy of an isolated system may be described by the sum of kinetic and potential energies, represented by Hamiltonian. 
where $p_{i}$ denotes a definite probability having the outcome $E_{i}$ in a specified maximal test for the quantum system. Since the internal energy (11) can be expressed as $U=U(\Theta, L)$, we can obtain the representation of the first law of quantum thermodynamics in terms of $\Theta$ 's and $L$ 's in $\mathcal{M}_{\Theta, L}$ (see below).

Let us consider a small change in the internal energy. Differentiating Equation (11) formally, we obtain

$$
d U=\sum_{i}\left(E_{i} d p_{i}+p_{i} d E_{i}\right)
$$

This indicates that the change in $U$ is originated from the two independent source, i.e., an outcome $E_{i}$ and a definite probability $p_{i}$. The two terms in the right-hand side of Equation (12) can be identified with the respective terms of Equation (10) for the expression of the first law of thermodynamics. The first term $\sum_{i} E_{i} d p_{i}$ is a consequence of the change in probabilities. We shall see that it corresponds to the heat flow into the system. This implies that the effect of heat transfer is to change the definite probabilities of the various outcome of $E_{i}$. The second term $\sum_{i} p_{i} d E_{i}$ relates to the change in the outcome $d E_{i}$. This term shows that change in the internal energy partially comes from the change in outcome. We shall show below that this corresponds to the work done on the system, i.e., $d^{\prime} W$. It must be emphasized that the validity of Equation (12) is ensured by Equations (13) and (19), i.e., existence of parameters, $\Theta$ and $L$, as will be shown shortly.

In order to understand that the second term corresponds to $d^{\prime} W$, we first note that the work done on the system is related to the variation of an external parameter (work coordinate) and the outcome should depend on the size (volume) of the system: $E_{i}=E_{i}(V)$. The work done on the system is due to the change of the volume of the system. Accordingly, without loss of generality we ascribe the change of the volume to the change of the work coordinate denoted by $L$ : the energy of state $i$,i.e., outcome $E_{i}$, which is the eigenvalue of the system's Hamiltonian $\mathscr{K}$, depends thus on the work coordinate $L$ : $E_{i}=E_{i}(L)$. This expression states that one can identify the work coordinate $L$ with the label of a maximal test introduced in the last section. Since $E_{i}$ is a function of $L$, we can write that the change in the outcome $E_{i}$ is expressed in terms of the work coordinate:

$$
d E_{i}=\frac{\partial E_{i}(L)}{\partial L} d L
$$

Then

$$
\sum_{i} p_{i} d E_{i}=\sum_{i} p_{i} \frac{\partial E_{i}}{\partial L} d L
$$

In Sec. 3, it was shown that $p_{i}=P(\Theta ; x=i)$. This indicates that $p_{i}$ is a function of $\Theta$. Thus we can write Equation (14) as

$$
\sum_{i} p_{i} d E_{i}=\sum_{i} \frac{\partial}{\partial L}\left(p_{i} E_{i}\right) d L=\frac{\partial}{\partial L} U d L
$$


A change in the internal energy of the system is generally related to a "force" defined by

$$
F \equiv-\frac{\partial U(L)}{\partial L},
$$

so that Equation (15) and hence Equation (14) can be expressed as

$$
\sum_{i} p_{i} d E_{i}=-F d L
$$

This indicates that the term $\sum_{i} p_{i} d E_{i}$ corresponds to the work $d^{\prime} W$ done on the system and it is generally expressed by ${ }^{4}$

$$
d^{\prime} W=\sum_{i} p_{i} d E_{i}(L)=-F d L
$$

where the definite probability $p_{i}$ can then be replaced by the probability function $P(\Theta ; x=i)$. It is important to notice that Equation (18) expresses the work $d^{\prime} W$ with respect to the change in $L$ (work coordinate) in a quasi-static process. Hence, the invariance of an external parameter $L$ implies $d^{\prime} W=0$. Thereby we have $d U=d^{\prime} Q$. Then the term $\sum_{i} E_{i} d p_{i}$ corresponds to the change in the internal energy of the system that occurs when no work is done; this is what we understand as heat flow.

It should be emphasized that the heat entering the system, $d^{\prime} Q$, is expressed in terms of the variation of $p_{i}=P(\Theta ; x=i)$ while the work done on the system, $d^{\prime} W$, is expressed in terms of the variation of $E_{i}(L)$. Since $p_{i}=P(\Theta ; x=i)$, we can write $d p_{i}$ as

$$
d p_{i}=\frac{\partial P(\Theta ; x=i)}{\partial \Theta} d \Theta,
$$

so that $d^{\prime} Q$ is expressed in terms of $\Theta$ :

$$
d^{\prime} Q=\sum_{i} E_{i} d p_{i}=\sum_{i} E_{i} \frac{\partial P(\Theta ; x=i)}{\partial \Theta} d \Theta .
$$

In general, unitary operator generated by the Hamiltonian of a system does not change the definite probability $p_{i}$. Therefore, the term $E_{i} d p_{i}$ representing the heat cannot be explained by the Hamiltonian itself. In the present theory, the effect of heat is expressed as a change in the definite probabilities having various outcomes $E_{i}$ in the maximal test. It should be noted that an adiabatic process is characterized by the case where $d p_{i}=0$ in the definition of heat, see Equation (20). After all, the representation for the heat in the present theory is consistent with a well-known adiabatic equivalence and the first law of thermodynamics for quantum systems can be represented by the elements in $\mathcal{M}_{\Theta, L}$ as seen in Equations (18) and (20).

As a consequence of the previous section (see the proof of $\mathbf{L} 1$ ) and the discussion above, the following corollaries, $\mathbf{C} 1$ and $\mathbf{C} 2$, can be drawn:

C1. The internal energy $U$ is specified by the parameter $\Theta$ and $L$ :

${ }^{4}$ We note that Equation (18) is a quantum analog of the expression $d^{\prime} W=-P d V$ in classical case. 


$$
U=U(\Theta, L) .
$$

Proof of $\mathrm{C} 1$. Once a maximal test is chosen for a fixed $L$, respective outcome $E_{i} \quad(i=1,2, \cdots)$ for the system is uniquely determined and the definite probability $p_{i}$ is then described as $P(\Theta ; x=i)$ by the proof of $\mathrm{L} 1$. Therefore, it is clear that the internal energy is specified by the parameters $\Theta$ and $L$.

We note that $\mathrm{C} 1$ states the internal energy $U$ can be specified by the parameters, $\Theta$ and $L$. We will omit $L$ in $U(\Theta, L)$ for simplicity when we consider a fixed $L$.

C2. $d^{\prime} Q=0$ implies the consequence of adiabatic equivalence.

Proof of C2. By the proof of L1, no change in the probability function implies that the absolute values of the expansion coefficients $\left|a_{i}\right|$ 's remain the same. This implies $p_{i}$ remains constant. Thus $d^{\prime} Q$ is equal to zero throughout the operation (during the process).

In the statement of $\mathbf{C 2}$, the consequence of adiabatic equivalence is as follows: if the system is isolated, the absolute values of the expansion coefficients $\left|a_{i}\right|$ 's would remain constant [11]. It should be noticed that the notion of heat arises only when the state (internal energy) of a system changes, where $d U \neq d^{\prime} W$. As in classical thermodynamics, heat in quantum systems is also defined as a form of energy movement. Once the internal energy of a quantum system is well defined, heat is also well defined. The notion of heat in quantum systems will be discussed further in a separate paper [12].

\subsection{The Second Law and Entropy}

In this section we will give a definition of entropy to describe the entropy principle (namely, the second law of thermodynamics) for quantum systems. The entropy principle states that the adiabatic accessibility of any two states is described by an entropy inequality. Here we should refer to the adiabatic process since the second law treated here is defined for the process. The process is characterized by $d^{\prime} Q=0$. This is ensured when $P(\Theta ; x)$ remains unchanged throughout the process (see $\mathrm{C} 1$ and argument below). In other words, adiabatic process is a process such that $P(\Theta ; x)$ remains unchanged. It should be noted that adiabatic process allows to change a value of $L$ since it only affects work $d^{\prime} W$. This is consistent with adiabatic process defined by Lieb and Yngvason [3].

Let us define an entropy function as a map from the set $\mathcal{M}_{\Theta, L}$ to a real number $R$ :

$$
S: \mathcal{M}_{\Theta, L} \mapsto R .
$$

We note that this general definition for entropy can describe all types of entropy functions including well known Boltzmann, Gibbs, and Shannon entropies. The entropy $S$ defined by the map (22) is clearly a state quantity 
and ensures that $S$ can be defined for all states in terms of $\Theta$.

In order to obtain a representation of the second law in terms of $\Theta$ for quantum systems, however, it must be shown that determining a parameter $\Theta$ as a state variable means to determine an internal energy $U$ as a state quantity. We have already shown that $U$ is specified by $\Theta$ 's and $L$ 's (i.e., the elements of $\mathcal{M}_{\Theta, L}$ ) as described in $\mathrm{C} 1$ and can be expressed by a function on $\mathcal{M}_{\Theta, L}$. Thus the following lemma (L2) is established:

L2. There exists $U$ specified by each element of $\mathcal{M}_{\Theta, L}$.

Proof of L2. Without loss of generality, one can consider a fixed maximal test, where outcome of the maximal test is uniquely determined: The internal energy is represented by $U=\sum_{i} p_{i} E_{i}=\sum_{i} P\left(\Theta_{\alpha} ; x=i\right) E_{i}$, whereby $\Theta_{\alpha}$ specifies the internal energy $U$. Therefore, $U$ can be labeled as $U_{\alpha}$. As in the same way, respective internal energies, $U_{\beta}, U_{\gamma}, \cdots$, can be specifically specified by $\Theta_{\beta}, \Theta_{\gamma}, \cdots$.

Since the existence of correspondence between an internal energy $U$ and a parameter $\Theta$ was established by L2, we can obtain one-to-one correspondence between $S(X)$ and $S(\Theta)$. This keeps consistency between an entropy function defined in the entropy principle and the statement (22). Put $U$ instead of $X$ in the statement (1) in Sec. 2 and we finally obtain a representation of the second law of quantum thermodynamics in terms of $\Theta$ :

$$
\Theta_{\alpha} \prec \Theta_{\alpha^{\prime}} \text { if and only if } S\left(\Theta_{\alpha}\right) \leq S\left(\Theta_{\alpha^{\prime}}\right) \text {. }
$$

This describes the entropy principle for quantum system. It should be noted that each $\Theta$ can depend on different values of $L$. Thus, Eq. (23) is able to give the information as to a complete structure in $\mathcal{M}_{\Theta, L}$, which enables us to compare $\Theta$ 's that depend on different values of $L$ 's in a context of adiabatic accessibility (recall that one can compare state vectors when $L$ 's are fixed). We should note that two quantum states represented by $\Theta_{\alpha}$ and $\Theta_{\beta}$ are adiabatic equivalent if and only if $S\left(\Theta_{\alpha}\right)=S\left(\Theta_{\beta}\right)$ for a fixed work coordinate $L$ (cf., A1 for classical case and the argument therein).

Now we consider the case corresponding to the statement (4) in Sec. 2. This states that the second law can refer to a possible adiabatic transition of a compound system consisting of system A and B when the compound system is thermally isolated from any other environment. From L2, we can immediately obtain the following relation: For a given arbitrary pair of states represented by $\left(\Theta_{\alpha}, \Theta_{\beta}\right)$ and $\left(\Theta_{\alpha^{\prime}}, \Theta_{\beta^{\prime}}\right)$, the following relation holds:

$$
\left(\Theta_{\alpha}, \Theta_{\beta}\right) \prec\left(\Theta_{\alpha^{\prime}}, \Theta_{\beta^{\prime}}\right) \text { if and only if } S\left(\Theta_{\alpha}\right)+S\left(\Theta_{\beta}\right) \leq S\left(\Theta_{\alpha^{\prime}}\right)+S\left(\Theta_{\beta^{\prime}}\right) \text {, }
$$

where $\Theta_{\mathrm{j}}, \mathrm{j}=\alpha, \beta, \cdots$, is the element of the state space $\mathcal{M}_{\Theta} \times \mathcal{M}_{\Theta}$. It should be noted that each $\Theta$ can depend on different values of $L$. This is the entropy 
principle expressed in terms of $\Theta$ 's for a quantum compound system. The statement (24) means that $\left(\Theta_{\alpha^{\prime}}, \Theta_{\beta^{\prime}}\right)$ is adiabatically accessible from $\left(\Theta_{\alpha}, \Theta_{\beta}\right)$. We note that this statement is useful when one considers the heat transfer between quantum systems, e.g., thermal contact. We will discuss thermal contact in a separate paper [12].

Now we consider a relation between parameter $\Theta$ and thermodynamic temperature $T$. We start with introducing thermodynamic temperature $T$ that is defined as a partial derivative of entropy $S$ with respect to $U$ :

$$
\frac{1}{T}:=\left.\frac{\partial S(U, V)}{\partial U}\right|_{V},
$$

where $U$ and $V$ denote the internal energy and volume (work coordinate) of a system, respectively [2] [3]. In the definition (25), differentiability of the entropy function $S$ may be ensured by a concavity of the entropy function. ${ }^{5}$

Here, we introduce a corollary C3:

C3. There exists a one-to-one correspondence between $T$ and $\Theta$.

Proof of C3. From the definition of thermodynamic temperature (25), the value of $T$ is defined for the respective values of $U$ owing to a uniqueness of the differential coefficient of a concave function $S$. Let $E$ be a value of internal energy $U(=E)$. By $\mathbf{C 1}$, the value $E$ has one-to-one correspondence with the value of $\Theta$. Therefore, thermodynamic temperature $T$ can be determined by the parameter $\Theta$ in $\mathcal{M}_{\Theta}$ introduced in Sec.3.

This corollary establishes the consistency between the parameter $\Theta$ and thermodynamic temperature $T$. Let us consider the partial derivative in the right-hand side of Equation (25). We note that a value of $T$ is determined by $\Theta$ and $L$. This implies that an internal energy plays the same role as temperature in quantum system (see $\mathbf{C} 1$ ). This agrees with the proposition of Bender et al. [9]. Equation (25) is useful to obtain the relation between thermodynamic temperature and quantum states characterized by $\Theta$. This is also useful to obtain a structure in the thermodynamic state space $\mathcal{M}_{\Theta}$.

In this section, we have established the representation of the second law in terms of the entropy $S$ for quantum systems as a function of parameter $\Theta$, so that one can define thermodynamic temperature $T$ as a function of a state parameter $\Theta: T=T(\Theta)$.

\subsection{The Third Law}

Let us briefly discuss the third law of thermodynamics for quantum systems. The third law requires quantum states [1]. Therefore, it must take into account the probabilistic nature of quantum theory. The probability function $P(\Theta)$ is

${ }^{5} \mathrm{~A}$ relation between thermodynamic temperature and concavity of the entropy function is discussed by Lieb and Yngvason [3]. 
found from probability amplitudes (see L1 in Sec. 3). Accordingly we can obtain the representation of the third law: The entropy $S$ is equal to zero only when the probability function satisfies the condition:

$$
P(\Theta ; x=i)=1 \text { for arbitrary } i \text {. }
$$

At the absolute zero temperature, one can expect a state of quantum system being in a single state such as

$$
|\Psi\rangle=a_{i}|i\rangle \text { for arbitrary } i .
$$

The single state here means that only one outcome is obtained with a probability one by maximal tests.

\section{Results and Discussion}

In this paper, we presented a representation of the basic laws in thermodynamics for quantum system in a pure state and investigated a relationship between thermodynamics and quantum theory. We obtained the representation of the zeroth, first, second and third laws in quantum thermodynamics, which affords the key to treat quantum system thermodynamically. In the derivation of the zeroth law that assures the existence of a property called "temperature" in classical thermodynamics, we proposed parameters $\Theta$ that characterize the equivalence law among state vectors, leading to the zeroth law of quantum thermodynamics. We showed that the existence of such parameters depends on the probabilistic nature of quantum theory (L1). It should be however noted that the parameters $\Theta$ 's introduced in our formulation make sense only when there exists the map $\mathcal{M}_{\Psi} \mapsto \mathcal{M}_{\Theta}$, i.e., $\mathcal{M}_{\Theta}$ is an injection of $\mathcal{M}_{\Psi}$. Therefore, in order to establish the representation of the zeroth law in terms of the elements in $\mathcal{M}_{\Theta}$, same maximal tests must be performed to obtain the corresponding elements of $\mathcal{M}_{\Theta}$ from the respective elements in $\mathcal{M}_{\Psi}$. Though this statement can refer only to the case where the work coordinates are fixed, the second law releases this restriction.

We established the representation of the first law, where the internal energy of quantum system as a state quantity is specified by the parameters in $\mathcal{M}_{\Theta, L}$ (C1). From the first law, when the state changes from $\Theta_{\alpha}$ to $\Theta_{\beta}$, heat entering the system is represented by $d^{\prime} Q=U\left(\Theta_{\beta}\right)-U\left(\Theta_{\alpha}\right)$ in the case where $d^{\prime} W=0$ (i.e., the work coordinate is fixed). Thus one can measure the quantity of heat in terms of $\Theta$ 's for quantum systems. By considering the adiabatic process ( $d^{\prime} Q=0$ ), one can also quantify the work done to the system in terms of $\Theta$ 's in the present theory. Since the term $d^{\prime} W$ is due to a shift of the outcomes caused by the variations of work coordinate, one can measure the work $d^{\prime} W$ in terms of the changes in the internal energy as $d^{\prime} W=U\left(\Theta_{\alpha}, L_{1}\right)-U\left(\Theta_{\alpha}, L\right)$ when we consider an adiabatic process, where $\Theta_{\alpha}$ remains constant. Here $U\left(\Theta_{\alpha}, L_{1}\right)$ describes the internal energy of the final state. This is useful to obtain the relation among $\left\{\mathcal{M}_{\Theta, L_{1}}, \mathcal{M}_{\Theta, L_{2}}, \cdots\right\}$ and the relation gives a structure in thermodynamic state space $\mathcal{M}_{\Theta, L}$. 
We also obtained the representation of the second law of thermodynamics for quantum system. The representation depends on L2 and assures the entropy principle. The representation of the second law (entropy principle) proposed here would give some insight into the structure (order relation) in $\mathcal{M}_{\Theta, L}$ for quantum systems. Therefore, this would afford one to investigate what thermodynamic structure exists in a state space describing thermodynamics of quantum systems in terms of those parameters in $\mathcal{M}_{\Theta, L}$.

The third law requires as a principle that entropy should be zero at the absolute zero temperature [1]. In our representation, entropy can take the value of zero when the state is described by $\left|\Psi^{\mathrm{j}}\right\rangle=b_{i j}|i\rangle$ (i.e., the state of the system is characterized by a single state for each ensemble $\mathrm{j}$ ). Since one can see the existence of a unique function of $\Theta$ that describes the thermodynamic temperature, there is no necessity of referring to an explicit form of the function. Let $|i\rangle$ be an energy eigenstate of the system. At the absolute zero temperature, the state is occupied from the lower states and the state $\left|\Psi^{\mathrm{j}}\right\rangle=b_{i j}|i\rangle$ should correspond to the lowest energy level $i$. Therefore, we required one more condition to the statement Equation (26), that is, the only state $i$ is reserved for the description of the lowest energy level. It should be noted that this requirement is consistent with Fermi and Bose statistics at the absolute zero temperature and the state is free from the type of Hamiltonian for quantum system, establishing the third law of quantum thermodynamics. By the third law along with the definition of entropy function (22), one can determine the absolute value of entropy.

Finally we consider the problem of thermal contact. This problem makes the notion of heat transfer much clear. Let us consider a compound system comprised of subsystem A and B whose initial states are characterized by $\left(\Theta_{\alpha}, \Theta_{\beta}\right)$, respectively. Without loss of generality we could use the same value of the work coordinate for both systems. The compound system as itself is isolated from any other environment. After thermal contact the state of each subsystem becomes $\left(\Theta_{\gamma}, \Theta_{\gamma}\right)$ due to the heat transfer between subsystems. For such a case, one can predict a possible value of $\Theta_{\gamma}$. Since the case satisfies the inequality (24), we could evaluate the values of $\Theta$ by obtaining the probability function $P(\Theta ; x)$. Therefore one can verify the order relation among $\Theta$ 's (i.e., whether $\left(\Theta_{\alpha}, \Theta_{\beta}\right) \prec\left(\Theta_{\gamma}, \Theta_{\gamma}\right)$ is true or not). In other words, by studying the order relation among parameters $\Theta$ 's through the study of respective entropies, thermodynamic structure hidden in quantum systems could be elucidated.

Now we discuss again the validity of the zeroth law (8) from a viewpoint of the thermal contact. If the subsystem A and B are in thermal equilibrium, then there is no heat transfer between them. Thus one can say $\Theta$ 's are the parameters which characterize respective thermal equilibrium states of subsystems A and B.

By applying the quantum thermodynamics presented in this paper, thermodynamic nature of quantum systems could be studied in all temperature 
without considering detailed mechanics of quantum systems. This is the advantage to utilize quantum thermodynamics presented in this paper. We hope that the present theory sheds light on further understanding a relationship between thermodynamics and quantum theory.

\section{Conflicts of Interest}

The authors declare no conflicts of interest regarding the publication of this paper.

\section{References}

[1] Fermi, E. (1956) Thermodynamics. Dover, New York.

[2] Callen, H.B. (1985) Thermodynamics and an Introduction to Thermostatics. 2nd Edition, Wiley, New York.

[3] Lieb, E.H. and Yngvason, J. (1999) The Physics and Mathematics of the Second Law of Thermodynamics. Physics Reports, 310, 1-96. https://doi.org/10.1016/S0370-1573(98)00082-9

[4] von Neumann, J.L. (1932) Mathematische Grundlagen der Quantenmechanik. Springer, Berlin. [Beyer, E.T. (Trans.) (1955) Mathematical Foundations of Quantum Mechanics. Princeton University Press, Princeton.]

[5] Peres, A. (1993) Quantum Theory: Concepts and Methods. Kluwer-Academic, Boston.

[6] Wehrl, A. (1978) General Properties of Entropy. Reviews of Modern Physics, 50, 221. https://doi.org/10.1103/RevModPhys.50.221

[7] Partovi, M.H. (1989) Quantum Thermodynamics. Physics Letters A, 137, 440-444. https://doi.org/10.1016/0375-9601(89)90221-1

[8] Scully, M.O., Zubairy, M.S., Agarwal, B.S. and Walther, H. (2003) Extracting Work from a Single Heat Bath via Vanishing Quantum Coherence. Science, 299, 862-864. https://doi.org/10.1126/science.1078955

[9] Bender, C.M., Brody, D.C. and Meister, B.K. (2000) Quantum Mechanical Carnot Engine. Journal of Physics A: Mathematical and General, 33, 4427-4436. https://doi.org/10.1088/0305-4470/33/24/302

[10] Isham, C.J. (1995) Lectures on Quantum Theory: Mathematical and Structural Foundations. Imperial College Press, London. https://doi.org/10.1142/p001

[11] Born, M. and Fock, V. (1928) Beweis des Adiabatensatzes. Zeitschrift für Physik, 51, 165-180. https://doi.org/10.1007/BF01343193

[12] Taira, H. and Suzuki, A. Representaion of Thermodynamic Laws in Quantum Systems II. (To be submitted) 